\title{
Renal Artery Stenosis: Diagnostic and Management Problems
}

Affa Kiysa Waafi ${ }^{1}$, Nur Samsu ${ }^{2}$

${ }^{1}$ Department of Internal Medicine, Faculty of Medicine, Universitas Brawijaya - dr. Saiful Anwar, General Hospital, Malang

2Division of Nephrology and Hypertension, Department of Internal Medicine, Faculty of Medicine, Universitas Brawijaya - dr. Saiful Anwar,

General Hospital, Malang

\section{ARTICLE INFO}

Corresponding Author:

Nur Samsu.

Division of Nephrology and

Hypertension, Department of

Internal Medicine, Faculty of

Medicine, Universitas Brawijaya -dr.

Saiful Anwar, General Hospital,

Malang

Email: nur_samsu.fk@ub.ac.id

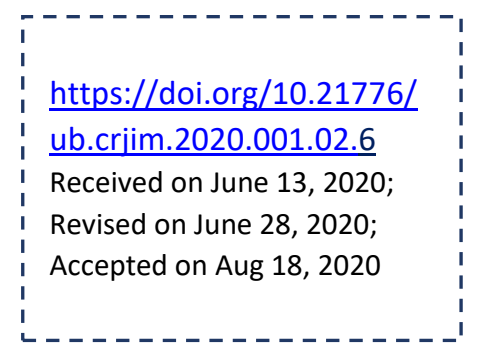

A B S T R A C T

Renal artery stenosis is one common clinical problem. It has wide spectrum of pathophysiology with 3 most common clinical syndromes, ischemic nephropathy, hypertension, and cardiac destabilization syndrome. Aim: To date there was not any specific diagnostic criteria for renal artery stenosis. Method: Clinicians only used some clinical syndromes to guide the diagnostic possibility of renal artery stenosis. RADUS as one sensitive and specific diagnostic method, still had some disadvantages. Results: it gives false negative results in $10-20 \%$ of patients due to confounding factors such as operator capability, obesity, or abdominal gas distribution. CTA and MRA was excellent, but possessed some risks for the patient. Therefore, CTA was mostly recommended in patient with the planning of revascularization. Management of renal artery stenosis was still debated between optimal medical management and revascularization because the complexities of mechanisms underlying the renal artery stenosis. Because of the complicated pathophysiology of renal artery stenosis, revascularization could not entirely improve renovascular hypertension and nephropathy. Revascularization offered best results in fibromuscular dysplasia, although procedure related complication was still high. Revascularization, even though it might have high success rate in atherosclerotic renal artery stenosis, but the incidence of re-stenosis was also fairly high. Conclusion: Overall, revascularization was recommended in FMD, but should only be preserved for atherosclerotic renal artery stenosis after the failure of optimal medical management.

Keywords: renal artery stenosis, diagnosis, medical management, revascularization

\section{N T RO D U C T I O N}

Renal artery stenosis becomes a
substantial clinical problem especially in
countries with poor resources. Generally,
renal artery stenosis was categorized into
atherosclerotic and non-atherosclerotic renal
artery stenosis. $90 \%$ of renal artery stenosis
was caused by atherosclerosis. Non-
atherosclerotic renal artery stenosis was
mostly caused by fibromuscular dysplasia

which composed $10 \%$ of all renal artery stenosis.[1,2] Diagnosis of renal artery stenosis is important because it could decrease renal blood flow and renal perfusion. Severe renal artery stenosis could activate the renin angiotensin aldosterone system. It would induce vasoconstriction and increase of peripheral artery resistance. ${ }^{[1,3,4]}$ Literatures suggest the relationship between renal artery stenosis and reno-vascular hypertension. 
Renal artery stenosis includes a wide spectrum of pathophysiology with 3 most common clinical syndromes, ischemic nephropathy, hypertension, and cardiac destabilization syndrome. ${ }^{[2]}$ To date, there are not any diagnostic criteria for renal artery stenosis. Some authors suggested clinical suspicion as a guide for diagnosing renal artery stenosis.[1,5] Renal artery duplex ultrasonography is recognized as a sensitive diagnostic method for renal artery stenosis, although there are still many confounding factors. It gave negative results in $10-20 \%$ of cases. Another imaging examination such as CTA (computerized tomography angiography) and MRA (magnetic resonance angiography), although highly sensitive and specific, both are expensive and inflict contrast and radiation exposure.[5,6]

Recently, studies indicated renal artery revascularization did not improve patients' outcomes in the aspects of renal function and cardiovascular outcomes. ${ }^{[7]}$ Angioplasty and stenting procedures offered excellent results for fibromuscular dysplasia, but evidence was not enough to prove benefit for blood pressure control and renal function improvement. Both procedures also possessed high prevalent risks and did not provide any prevention from cardiovascular risk. ${ }^{[8,9]}$ This review article explained about problems in the diagnosis and management of renal artery stenosis, especially in the setting of poor resources.

\section{Renal Vascularization and Glomerular Filtration Regulation}

Kidney worked as a unit of excretion. Beside excreting residual substances such as ammonia, urea, creatinine, and uric acid, it also excretes drugs and toxin. Kidney has another function as regulator of water and electrolyte equilibrium. It regulates the balance of acid base, calcium, phosphate, vitamin D metabolism, and red blood cell production.
Every nephron in kidney contains glomerulus, which function as ultrafiltration membrane, loop of Henle, distal renal tubules, and collective tubules.[4,8]

Blood flows through afferent arterioles to glomerular capillaries. In the glomerulus, capillaries rolled up densely, surrounded by Bowman capsules. Blood in glomerulus undergo ultrafiltration in glomerular basement membrane (GBM), which consisted of basal membrane of tubular epithelia and vascular endothelial cells. Majority of watersoluble substances will be filtrated through membrane. Filtration of glomerulus forms relatively large volume of plasma ultra-filtrate (about $120 \mathrm{ml} /$ minutes or $170 \mathrm{~L} /$ day). The ultra-filtrate will pass through tubules and undergo selective reabsorption. Distal end of glomerular capillaries merge and continue as efferent arterioles. Efferent arterioles will continue as second capillary networks (cortical peritubular capillaries or vasa recta medulla) around tubules. Efferent arterioles regulate hydrostatic pressure in both capillary networks. ${ }^{[4,8,9]}$

Hydrostatic pressure along capillary walls of glomerulus is the main force of glomerular filtration. Oncotic pressure inside capillary lumens plays the role of filtration barrier. Oncotic pressure was influenced by unfiltered plasma protein concentration. During filtration process, oncotic pressure will increase along glomerular capillaries with the decrease of filtration pressure. It reaches 0 $\mathrm{mmHg}$ in proximal of efferent arterioles. About $20 \%$ of plasma will be filtrated into Bowman capsules. Ratio of eGFR (estimated glomerular filtration rate) to renal blood flow determines filtration fraction. [4,9]

Although glomerular filtration rate was affected by renal arterial pressure, but the relationship was not linier. It was because of autoregulation of GFR. Glomerular filtration 
rate is the rate of blood that flows through nephrons after being filtered. GFR did not reflects general renal function and it could be affected by body surface area. GFR was calculated using some substances which were filtered entirely by glomerulus and were not reabsorbed by tubules, such as creatinine. Glomerular filtration pressure was constantly maintained under autoregulation process. It provided relatively constant glomerular filtration pressure under systemic blood pressure and cardiac output variation. ${ }^{[4,8]}$

Rate of filtration and reabsorption in nephron was controlled by hormonal signals and hemodynamic factors. Those components were also important in blood pressure regulation. Juxtaglomerular part of kidney secreted renin as response to decrease of afferent arteriolar pressure, sympathetic nerve stimulations, and sodium concentration changes in distal contorted tubules at macula densa. Renin production is the first step of angiotensin II production and release of aldosterone which will continue to induce systemic vasoconstriction and regulate extracellular volume. Renin cleaved angiotensinogen into angiotensin I, in which it would be converted by angiotensin converting enzyme into angiotensin II. Angiotensin II will return the pressure of glomerular perfusion rapidly by vasoconstriction of efferent arterioles inside kidney and through systemic vasoconstriction. Both conditions will induce increase of kidney perfusion. In the long term, angiotensin II will increase plasma volume through stimulation of aldosterone release in which it will induce sodium reabsorption through renal tubules. ${ }^{[8,10]}$

The increase of arteriolar afferent pressure will be accompanied by increase of urine output through the phenomenon of pressure diuresis. This condition will be reflected by renal urinary output curve or renal function curve. In artery pressure of 50 $\mathrm{mmHg}$, almost there is not any urinary output. In artery pressure about $100 \mathrm{mmHg}$, urinary output will be normal and in artery pressure of $200 \mathrm{mmHg}$, the urinary output will be 8 times than normal. Increase of arterial pressure also increases sodium output through the process of pressure natriuresis.[10]

\section{Epidemiology and Etiology of Renal Artery Stenosis}

Prevalence of renal artery stenosis in general population is relatively small. About 1$6 \%$ of patients with hypertension, was suspected to have element of renal artery stenosis. In population of patients that undergone coronary arteriography, the prevalence increased significantly to $>20 \%$. In a study of 1302 patients which undergone coronary arteriography, renal artery stenosis occurred in $15 \%$ of participants.[2,11]

Renal artery stenosis is a spectrum of conditions with different pathophysiology, so it needs comprehensive approach of diagnosis and management. Generally, it is categorized as atherosclerotic and non-atherosclerotic renal artery stenosis. About $90 \%$ of renal artery stenosis was caused by atherosclerosis. Atherosclerosis often affects $1 / 3$ proximal of renal artery stenosis, includes perirenal aorta and ostium. Non-atherosclerotic renal artery stenosis includes a large number of etiology such as fibromuscular dysplasia, aneurysm, arteriovenous fistulas, vasculitis, neurofibromatosis, trauma, emboli, congenital band, radiation therapy, and vascular dissection. Fibromuscular dysplasia is the second most common cause of renal artery stenosis, which about $10 \%$ of all renal artery stenosis. Although it was similar to vasculitis, definitive cause of fibromuscular dysplasia is still not known. ${ }^{[1,2]}$ Table 1 presents some causes of renal artery stenosis.

Table 1. Causes of Renal Artery Stenosis ${ }^{[1]}$

\begin{tabular}{cc}
\hline Classification & Causes \\
\hline Atherosclerotic & Atherosclerosis
\end{tabular}




$\begin{array}{ll}\text { Non- } & \text { Fibromuscular dysplasia } \\ \text { Atherosclerotic } & \text { Nephroangiosclerosis } \\ & \text { (Hypertensive injury) } \\ & \text { Diabetic nephropathy (small } \\ & \text { vessels) } \\ & \text { Renal thromboembolic disease } \\ & \text { Atheroembolic renal disease } \\ & \text { Aortorenal dissection } \\ & \text { Renal artery vasculitis } \\ & \text { Trauma } \\ & \text { Neurofibromatosis } \\ & \text { Thromboangiitis obliterans } \\ \text { Scleroderma } \\ \text { Extrinsic compression }\end{array}$

Renal artery stenosis seldom generated major hemodynamic effects. Atherosclerotic renal artery stenosis often related to renovascular hypertension and nephropathy. Atherosclerotic renal artery stenosis was relatively common in population $(6.8 \%$ in population $>65$ y.o) and the prevalence continues to increase with the increase of age. It was also commonly found in patient with other vascular diseases such as coronary artery disease (18-23\%) and/or peripheral artery disease and lower extremity artery disease (>30\%). More than 50\% atherosclerotic renal artery stenosis will worsen in 5 years if it leaved without any treatment. ${ }^{[2,4]}$

Fibromuscular dysplasia was reported in $3-5 \%$ of potential kidney donor candidate without any hypertensions. But this condition was also reported in young patient with hypertension (with age about 15-50 y.o.), especially women. FMD rarely disrupts kidney function but sometimes FMD causes total occlusion and renal artery aneurysm. ${ }^{[4,12]}$

\section{Pathophysiology of Renal Artery Stenosis and Clinical Manifestation}

Classically, renovascular diseases were classified into two broad groups, hypertension and nephropathy, in which both of them are related to renal artery stenosis. ${ }^{1}$ Severe unilateral renal artery stenosis would activate renin angiotensin aldosterone system (RAAS).
It actually increased vasoconstriction and peripheral arterial resistance. If the contralateral kidney was normal without any stenosis, the effect of volume expansion would still be restricted by sodium and water excretion through contralateral kidney. But in the condition of bilateral renal artery stenosis, peripheral arterial resistance and blood volume continued to increase which lead to high blood pressure and overload condition. ${ }^{[3,4]}$

Renin angiotensin aldosterone system has role in maintaining vascular tones, watersodium balance, and cardiac function through the interaction of sympathetic nervous system and some other hormones. The system will be activated in the condition of hypotension, decrease intravascular volume, hyponatremia, hypokalemia, and chloride changes. The system detects changes of intravascular volume through changes in $\mathrm{Na}-\mathrm{K}-\mathrm{Cl}$ cotransporter at macula densa in kidney. Activated RAAS induces release of renin from juxtaglomerular apparatus inside kidney. Renin cleaves angiotensinogen into angiotensin I. Angiotensin I will be cleaved by angiotensin converting enzyme into angiotensin II. Angiotensin II binds to AT1 receptor in the kidney. The binding induces cascades that will eventually causes vasoconstriction of efferent arterioles, aldosterone stimulation, and increase sodium reabsorption.[1,13]

Renal artery stenosis progresses in a long period of time. It allows both kidneys to slowly adapt to decrease of blood flow through autoregulation. ${ }^{[3]}$ Activated renin angiotensin aldosterone system aims to increase renal perfusion through systemic blood pressure rise. Beside vasoconstriction, water and sodium retention, aldosterone secretion, and sympathetic nerve activation, activated RAAS also induces vascular remodeling and 
hypertension. Vasoconstriction in efferent arterioles of glomerulus will maintain glomerular filtration function in short time period and prevent kidneys from hypoxia. But in long period of time, RAAS will induce ischemic nephropathy in stenotic kidney, nephro-sclerotic hypertension in non-stenotic contralateral kidney, and glomerulosclerosis and interstitial fibrosis in both kidneys. Glomerulosclerosis and tubule-interstitial fibrosis was presumably due to increase of angiotensin II which related to increase of proinflammatory cytokine, which in turn increase inflammatory and pro-fibrogenic pathway. In the condition of severe bilateral renal artery stenosis or stenosis in one functional kidney, kidney will losses its capacity to maintain balance of sodium and fails to induce pressure natriuresis against blood pressure increase. This will lead to inappropriate peripheral vasoconstriction with significant increase of afterload, which can lead to myocardial ischemia and heart failure. $[1,5,9]$

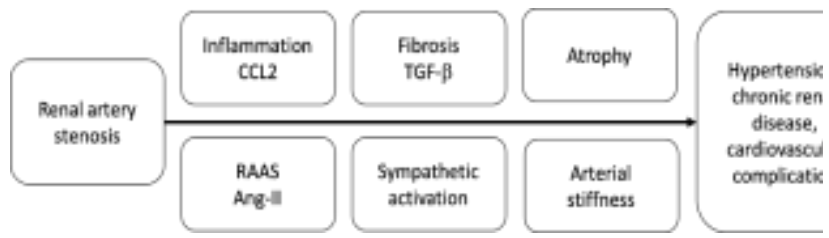

Figure 1. Pathophysiology of renal artery stenosis [7]

In an experimental study, significant hemodynamic changes that causes hypertension occurred in $75-80 \%$ of lumen occlusion. But critical point for lumen occlusion in human still could not be determined. Generally, stenosis for $>50 \%$ or $70 \%$ of lumen was defined as significant renal artery stenosis. [3]

Hypertension in renal artery stenosis was also caused by some other mechanisms, such as increase sympathetic tones, endothelial dysfunction, oxidative stress, ischemic nephropathy, and end organ damage.[3] Overall, renal artery stenosis will cause systemic hypertension which indicated by dependencies to angiotensin in initial stage, wide range of blood pressure variation, losses of circadian blood pressure rhythm, and acceleration of end organ target damage, such as left ventricular hypertrophy and renal fibrosis.[4,13]

Some recent studies oppose the idea of RAAS as the main factor played role in renovascular hypertension in renal artery stenosis. Studies using imaging technique showed that kidneys with arterial stenosis were not entirely hypoxic and even the stenotic kidney received higher blood flow than non-stenotic kidney. Studies also observed that kidneys had adaptation capacity to significant decrease of renal artery so they can maintain their oxygen capacity. Systemic RAAS activation tend to be temporarily but progress of kidney disease and cardiovascular dysfunction could happen without any persistent increase of angiotensin II. It indicated that even though systemic RAAS activation only last for a short period of time, but intra-renal RAAS activation was persistent because kidney could produce all elements needed in RAAS activation. Persistent RAAS activation occurred in stenotic kidney, but not in contralateral kidney. ${ }^{[7]}$

Fibromuscular dysplasia (FMD) is often found in pre-menopausal women with age between 15-50 years old and it closely related to history of hypertension and smoking. FMD patients generally had good clinical condition with low cardiovascular risk. Hypertension in FMD did not related to obesity, oral contraception, and family history.[1] Histologically, FMD includes intimal, medial, and adventitial layer of renal artery, but 90\% was found in medial layer. In angiography, FMD was described as beads-on-a-string because the contrast filled the aneurysm parts alongside renal artery. Most FMD causes 
stenosis at $2 / 3$ of distal renal artery. But it could also involve carotid and vertebral arteries. FMD had good prognosis and did not progress into total occlusion. But in $2 / 3$ of patient, FMD is bilateral and causes renovascular hypertension, but rarely become nephropathy. ${ }^{[2,9]}$

Pathologically, FMD had 3 subtypes: $[2,4,6]$

a. Intimal fibroplasia: It encompasses 5$10 \%$ of FMD and is often found in children and young adult. But it is not always found in women. Histologic examination showed collagen fibres deposit circularly at intimal layer which produced smooth tubular stenosis.

b. Medial fibroplasia: It is the most common pathological finding in FMD (75-80\%), which commonly found in middle aged women. There was the thinning of intimal and medial layer with losses of elastic lamina, which lead to aneurysm formation. The area of aneurysm was interspersed with local areas of fibrosis at medial layer. In angiography examination, those areas were referred as string of beads. One third of arteries were usually normal and $40 \%$ cases were bilateral.

c. Perimedia fibroplasia: It is found in about $10 \%$ of FMD. Fibrosis tissue was formed in the outer layer of medial muscle layer. It causes severe stenosis without aneurysm.

Atherosclerotic renal artery stenosis often happened in older individuals, in which $7 \%$ was diagnosed in patient $>65$ years old and $60 \%$ was diagnosed in patient with history of hypertension, coronary artery disease, peripheral artery disease, and renal insufficiency. Atherosclerotic renal artery stenosis commonly involved artery ostia, $1 / 3$ proximal of renal artery, and nearby aorta. Atherosclerotic renal artery stenosis related to renovascular hypertension and nephropathy.[1]

Renovascular hypertension in renal artery stenosis typically arise in young age and worsened with increasing age. It could also manifest as accelerated hypertension or resistant hypertension. Resistant hypertension is defined as uncontrolled hypertension despite consumption of 3 or more anti hypertension drugs including diuretics, or malignant hypertension with end organ damage.[8] Clinically, there could be some abdominal systolic or diastolic bruit and also hypokalemia in renovascular hypertension. Most common acute cardiovascular manifestation in renovascular hypertension includes flash pulmonary edema, which is not caused by any other coronary arterial diseases. Other cardiovascular manifestation includes hypertension related to acute coronary syndrome, aortic dissection, transient cerebral ischemia, stroke, intracranial hemorrhage, encephalopathy, and papil edema.[12]

Renal manifestation of renal artery stenosis could include acute renal failure which was characterized by increase of creatinine level after consumption of ACEinhibitor or ARB. Acute renal failure typically happened in 10-14 days after first drug consumption, but occasionally it happened randomly. Although acute renal failure induced by ACE-inhibitor or ARB was considered as classic signs of renal artery stenosis, but it was not a sensitive nor specific indication for renal artery stenosis. Another renal manifestation is unreasonable chronic renal failure, asymmetrical renal size, and renal atrophy. About 5-15\% of dialysis needed chronic ischemic nephropathy was estimated to be caused by renal artery stenosis.[1,12] 


\section{Diagnostic Criteria for Renal Artery Stenosis}

Renal artery stenosis is a general term for any vascular lesion which induced constriction of renal artery lumen and actually decrease renal blood flow. Two most common etiologies of renal artery stenosis are fibromuscular dysplasia and atherosclerotic renal artery stenosis. Atherosclerotic renal artery stenosis is typically accompanied by atherosclerosis in other arteries. Renal artery stenosis creates a wide spectrum of pathophysiology, with 3 main clinical syndromes: ischemic nephropathy, hypertension, and cardiac destabilization syndrome.[2]

Diagnostic criteria for renal artery stenosis is not yet established, but there are some clinical conditions that can guide into the possibility of renal artery stenosis:[1,5]

- Onset of hypertension $<30$ y.o. or severe hypertension in age $>55$ y.o.

- Accelerated, resistant, or malignant hypertension

- Renal atrophy without any defined causes or renal size discrepancies $>1.5 \mathrm{~cm}$

- Flash pulmonary edema without any definitive causes

- Unreasonable renal dysfunction, even need a renal replacement therapy

- New azotemia or worsened of renal function after ACE inhibitor or ARB consumption

- Multi-vessels coronary artery disease or peripheral artery disease

- Congestive heart failure or refractory angina without any possible causes

Patient with clinical suspicion of renal artery stenosis could have sent for diagnostic evaluation. Physical examination sometimes only gave limited clues such as systolic/diastolic abdominal bruit which radiated into flank area.[5] Prevalence of abdominal bruit was only about 78-87\% even in patient with established renal artery stenosis. Systolic and diastolic bruit had sensitivity about 39\% and specificity about 99\%. Epigastric bruit or flank bruit had sensitivity about $63 \%$ and specificity about $90 \% \cdot{ }^{[6]}$

Biochemical blood analysis indicated renal dysfunction with increase of creatinine level in serum and increase of plasma renin activity. Sometimes, renal dysfunction also caused hypokalemia due to hyperaldosteronism. ${ }^{[8]}$ Urinary analysis showed increase urine concentration with decrease of sodium concentration, especially in kidney with ischemia. Estimated GFR will also lower in ischemic kidney.[9]

Some diagnostic evaluation for renal artery stenosis includes captopril-stimulated nuclear renal flow, magnetic resonance angiography (MRA), and computerized tomography angiography (CTA). Captopril stimulated nuclear renal flow was used for diagnosing renal artery stenosis in patient with normal renal function and considered as one effective diagnostic method. But its accuracy decreased in patient with bilateral renal artery stenosis.[1]

Table 2. Classification of Renal Artery Stenosis according to Angiography Results ${ }^{[5]}$

\begin{tabular}{|c|c|c|}
\hline $\begin{array}{l}\text { Angiograp } \\
\text { hic stenosis } \\
\text { severitya }\end{array}$ & Physiologic testing & Significance \\
\hline$<50 \%$ & None & Mild \\
\hline $50-70 \%$ & None & Indeterminate \\
\hline $50-70 \%$ with & $\begin{array}{l}\text { Resting mean } \\
\text { pressure gradient } \\
\quad>10 \mathrm{mmHg}\end{array}$ & Significant \\
\hline $50-70 \%$ with & $\begin{array}{c}\text { Systolic hyperemic } \\
\text { pressure gradient } \\
>20 \mathrm{mmHg}\end{array}$ & Significant \\
\hline $50-70 \%$ with & Renal $\mathrm{Pd} / \mathrm{Pa} \leq 0.8^{\mathrm{c}}$ & Significant \\
\hline$\geq 70 \%$ & None & Significant \\
\hline \multicolumn{3}{|c|}{$\begin{array}{l}\text { Visual estimation } \\
\text { 'Translesional gradient measured with a non-obstructive catheter, i.e. } \leq 4 \\
\text { Fr or with } 0.014 \text {-in pressure wire (Pd/Pa) } \\
\text { cHyperemia may be induced with intrarenal bolus of papaverine } 30 \mathrm{mg} \\
\text { or dopamine at } 50 \mu \mathrm{g} / \mathrm{kg}\end{array}$} \\
\hline $\begin{array}{r}\text { Renal } \\
\text { (RADUS) is }\end{array}$ & $\begin{array}{l}\text { tery duplex } \\
\text { of the sensit }\end{array}$ & $\begin{array}{l}\text { asonography } \\
\text { and specific } \\
\text { osis. It is not }\end{array}$ \\
\hline
\end{tabular}


expensive and can be repeated without any large risk. RADUS has sensitivity range about 84-98\% and specificity range about $97-99 \%$ according to studies from Taylor, et al. ${ }^{[14]}$ and Olin, et al.[15] RADUS defined renal artery stenosis through the evaluation of ratio between peak velocity of renal artery systolic blood flow to peak velocity of aorta. But ultrasound has some limitation such as it depends on body habitus, bowel gas around kidney sometimes obscure kidney image, and the quality of examination depends on operator. To evaluate nephropathy in RADUS, clinician can use renal resistive index (RI) which is the evaluation of arterial flow resistance in the renal vascular networks and it is calculated using Doppler arterial pulsation. RI value $>0.8$ is considered significant for severe renal parenchymal disease.[1,5] Some other criteria evaluate renal artery stenosis using peak systolic velocity (PSV), renal aortic ratio (RAR), and acceleration time (AT) in RADUS. PSV value $>180 \mathrm{~cm} / \mathrm{s}$ is the most accurate diagnostic value for renal artery stenosis. Hemodynamic effect due to lumen occlusion such as changes of pressure trans-lesion or changes of flow trans-lesion, will be difficult to detect unless the occlusion is critical (occlusion of $70-80 \%$ of lumen).[6,15]

Some axial imaging techniques such as CTA and MRA, also have high sensitivity and specificity for diagnosing renal artery stenosis, but with much higher cost. Although CTA has sensitivity about $94 \%$ and specificity about 93\%, CTA also possess high risk of contrast and radiation exposure.[5,6] MRA uses nonionic contrast and does not use radiation, but MRA is not recommended for only estimating the degree of severity. MRA has $90 \%$ of sensitivity and $94 \%$ of specificity. Patient with renal dysfunction has the risk of contrast induced nephropathy because of ionized contrast in CTA. Gadolinium contrast in MRA can induce nephrogenic systemic fibrosis.[16] Invasive angiography may be indicated in patient with inconclusive results of imaging and clinical manifestation of renal artery stenosis, especially in patient with indication of intervention and revascularization.

In his review, Gottam, et al.[1] mentioned, with the sophistication of technology, some studies showed significant increase of sensitivity and specificity of CTA and MRA for renal artery stenosis. Study by Vasbinder, et al.[16] showed MRA sensitivity about $62 \%$, CTA sensitivity about 64\%, MRA specificity about 84\%, and CTA specificity about 94\%.[16] In the studies by Postma et ${ }^{17}$ and Williams, et al.[18] CTA showed sensitivity about 98\% with specificity about $94 \%$ and MRA could show sensitivity as high as $100 \%$ and specificity about $96 \%$.

Renal angiography is the golden standard for invasive diagnostic evaluation for significant renal artery stenosis especially in patient with hemodynamic changes. Degree of stenosis was classified according to Table 2, but it did not accurately reflect clinically significant hemodynamic changes. Stenosis $>70 \%$ was considered severe and significant hemodynamically. Stenosis between $50-70 \%$ might not be significant hemodynamically and it indicated repeated evaluation. Consensus and some experimental studies classified severe hemodynamic dysfunction if the average trans-lesion gradient was $>10 \mathrm{mmHg}$, peak hyperemic systolic gradient pressure was $>20 \mathrm{mmHg}$, or renal fraction flow reserve $($ FFR $) \leq 0.8 .[5,18]$

\section{Management of Renal Artery Stenosis}

The main objective of renal artery stenosis management is to prevent decrease of renal function and reduce water and sodium overload. Most patient with renal artery 
stenosis had increase of mortality due to increase of cardiovascular dysfunction. Clinician had to ensure modification of changeable cardiovascular risk beside optimal management of renal artery stenosis.[9] Medical management is the main therapy of renal artery stenosis especially in atherosclerotic condition, in which patient was given oral anti hypertension, statin, and low dose aspilet.[8] Some studies also suggest diet, exercise and physical activity, and controlling other risk factors such as smoking and alcohol.[6]

Conservative or medical management is indicated for patient with RI $>80$, pulse pressure $>100$, and severe nephropathy with urine protein excretion $>1 \mathrm{~g} /$ day, creatinine clearance $<40 \mathrm{ml} /$ minute, hyperuricemia, and hypertension in nighttime. Some authors suggest pulse pressure $>70$.[1] Hypertension could be controlled with ACE inhibitor. But ACE-inhibitor might decrease renal perfusion which could be significant in post stenotic level. ACE inhibitor might also cause acute kidney injury in patient with hemodynamically significant bilateral renal artery stenosis. ${ }^{[9]}$ Beside ACE inhibitor, other anti-hypertension which could be recommended is angiotensin receptor blocker (ARB).[7]

When there is failure of conservative therapy in which the blood pressure did not come down, and accompanied by recurrent flash pulmonary edema, worsened azotemia, with evidence of significant renal artery stenosis, patient could be considered to be sent for revascularization. Revascularization should also consider about patient's age and pulse pressure. Optimal outcome of revascularization decreases with increasing age. ${ }^{[1]}$ Patient with pulse pressure $<70$ might still gain benefit from revascularization especially for blood pressure control with about $97 \%$ of sensitivity and specificity. Improvement of renal function has $80 \%$ sensitivity and $88 \%$ specificity. Revascularization in patient with pulse pressure $>100$ would even worsened blood pressure control with sensitivity about $73 \%$ and specificity about 99\%. It would also decrease renal function with sensitivity about $84 \%$ and specificity about $95 \% .{ }^{[9,19]}$

The main objective for revascularization is to improve blood pressure control, prevent progressive ischemic nephropathy, and improve heart failure, chronic angina or flash pulmonary edema. Revascularization is only indicated in hemodynamically significant renal artery stenosis. Table 3 shows some indications for revascularize-ation in renal artery stenosis.[5,9]

Table 3. Indications of revascularization in renal artery stenosis ${ }^{[5,9]}$

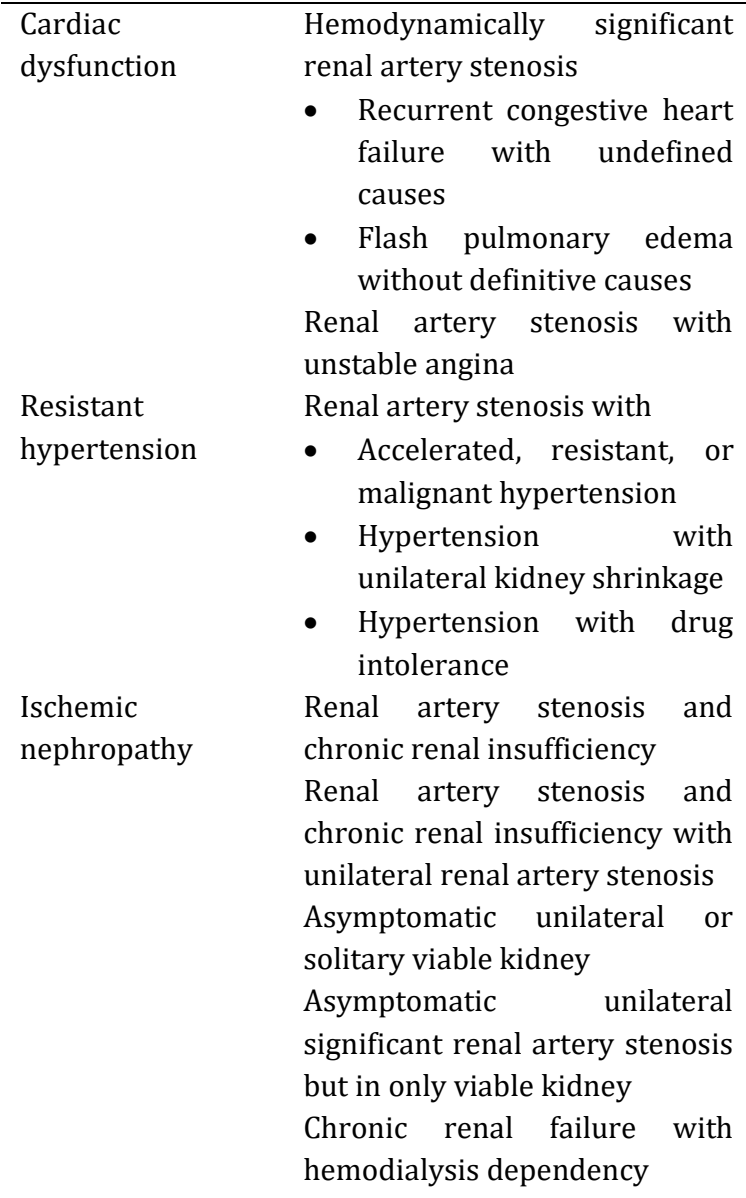


Progressive renal failure without definitive causes

Main recommendation for FMD revascularization is balloon angioplasty. Some studies revealed successful outcome of balloon angioplasty for FMD with nearly 100\% success rate with re-stenosis for only $<10 \%$ in 10 years. Angioplasty was proven to be more effective if the FMD was localized in major renal artery than diffused FMD. Some FMD cases were accompanied by aneurysm at renal artery in which if the aneurysm had big enough, it could be managed by graft stenting, occlusion with coil, or surgical reconstruction. ${ }^{[1,12]}$

\section{Diagnostic and Management Problem in Renal Artery Stenosis}

Fibromuscular dysplasia had some challenges in diagnostic process because although many patients showed symptoms and signs of resistant hypertension, but also many patients did not indicate any abnormalities in the physical examination and routine laboratory examination. Even RADUS was considered to be sensitive for diagnosing of renal artery stenosis, RADUS could produce false negative results in $10-20 \%$ of patient because of some confounding factors such as operator capability, obesity, or gas distribution at abdomen. ${ }^{[12,14]}$ Other imaging technique such as CTA and MRA were sensitive and specific, but very expensive. CTA also poses risk of exposure to ionized contrast and radiation. MRA contrast, gadolinium, gives the risk of nephrogenic systemic fibrosis.[5,6] Therefore CTA was mostly recommended in patient with planning of revascularization.

Some doubts were proposed regarding medical management for renal artery stenosis. Aggressive blood pressure control was considered to aggravate renal dysfunction, even though generally, blood pressure control could improve renal function with protecting contralateral kidney. Patient with medical management still had some risks to develop progressive renal dysfunction and cardiovascular events. Some studies observed inflammation reducing therapy may offer some benefit for some patients with chronic renal failure with renal artery stenosis.[7]

Best result of revascularization was obtained in non-atherosclerotic FMD in which stenosis correction often improve blood pressure control and renal function. ASTRAL study revealed angioplasty and stenting may give some benefit for atherosclerotic renal artery stenosis but the evidence was still scarce regarding improvement of blood pressure control and renal function. Angioplasty and stenting, on other side, also had procedural risks such as renal artery occlusion, renal infarct, and atheroembolism. ${ }^{[8,23]}$ Even $30 \%$ of renal intervention had complication because of atheroembolism. Recent studies mentioned renal artery stenting did not bring any significant benefit for preventing comorbidities such as cardiovascular event, renal disease progression, or the need of renal replacement therapy.[9,19]

According to Tegtmeyer, et al.[25] angioplasty in FMD, although had better prognosis than atherosclerotic renal artery stenosis, only offered overall recovery rate about $36 \%$ with post-operative recovery rate about $54 \%$. Re-stenosis rate for FMD was $20 \%$. Another recent study in more than 2000 patients, which recovery was defined as blood pressure $<140 / 90 \mathrm{mmHg}$ without any oral hypertension drugs, it was found that blood pressure outcome was significantly related with age.[28] Long term blood pressure control in FMD was about 93\%. Improvement or stabilization of renal function was observed in about $92 \%$. But complication of intervention 
procedure in FMD was as high as $12 \%$ after angioplasty and $17 \%$ after surgery. Major complication was observed in $6 \%$ cases after angioplasty and $17 \%$ cases after surgery. Besides, medical management with oral anti hypertension was considered effective in FMD in which clinician seldom attempted intervention management. ${ }^{[9]}$ But some authors found blood pressure control in FMD after revascularization was as high as $79 \%$ and $65 \%$ patient had successfully maintain blood pressure control until 8 years after revascularization. ${ }^{[1,29]}$

Angioplasty with stent placement was more recommended for atherosclerotic renal artery stenosis to prevent elastic recoil, minimalize arterial dissection, and maximize lumen widening. Successful outcome of angioplasty with stent placement in atherosclerotic renal artery stenosis, although could be as high as $95-100 \%$, but re-stenosis rate was also as high as $10-30 \%$ in 1 -year period.[1] Procedural related complication was also high which included arterial dissection, atheroembolism, and renal dysfunction. Even with newest techniques such as catheter-incatheter and no-touch technique to minimalize contact between guiding catheter with atherosclerotic plaque, but renal embolism was found in $60 \%$ of cases and became the main major factor for worsening renal function. ${ }^{[30,31]}$

There were three randomized clinical studies which compared angioplasty with medical management for blood pressure control in patient with renal artery stenosis. But none of those studies showed any significant differences in systolic blood pressure between 2 groups. Although DRASTIC study showed significant blood pressure improvement in angioplasty group than medical management group (68\% vs $38 \%$ ) and angioplasty group showed fewer worsening of blood pressure control (9\% vs $33 \%)$ and fewer renal artery occlusion (0\% vs 16\%) in 12 months. ${ }^{[29]}$ But to date, there was not any study comparing medical management with angioplasty and stent placement because recently stent placement had become standard therapy. Stent placement in renal artery stenosis improved technical outcome and clinical outcome in long term period, compared to angioplasty alone, especially in ostial stenosis ( $80 \%$ of lumen). In a meta-analysis of 1.322 patients, stent placement had better technical outcome and lower re-stenosis rate when was compared to angioplasty alone ( $98 \%$ vs $77 \%$ and $17 \%$ vs $26 \%$ respectively). It also offered higher recovery rate.[28]

Stent placement in renal artery stenosis generally related to systolic and diastolic blood pressure improvement and decreased the need of oral anti hypertension. Even blood pressure improvement could be observed in 24 hours after successful stent placement and maintained for 24 months. ASPIRE-2 study[26] evaluated safety and efficacy of balloon expandable stent placement after failure of angioplasty. It revealed re-stenosis in $17.4 \%$ of participants. Some predictors for re-stenosis in the study was diabetes, pre-procedural small diameter of vessels, and small minimum diameter of vessel after procedure. But this study also found the increase of average creatinine level from $1.36 \pm 0.52 \mathrm{mg} / \mathrm{dl}$ in baseline to $1.40 \pm 0.61 \mathrm{mg} / \mathrm{dl}$ in 9 months and became $1.46 \pm 0.81 \mathrm{mg} / \mathrm{dl}$ in 24 months. In a subgroup of patient with baseline creatinine level $1.46 \pm 0.81 \mathrm{mg} / \mathrm{dl}$, there was increase of creatinine to $1.46 \pm 0.81 \mathrm{mg} / \mathrm{dl}$ in 9 months and $1.46 \pm 0.81 \mathrm{mg} / \mathrm{dl}$ in 24 months. In 24 months, $7.5 \%$ of patient with abnormal renal function at baseline, had worsening of renal function although there were not any patients 
requiring permanent nor temporary hemodialysis.

CORAL study [28] compared optimal medical management with angioplasty and stent placement in patient with hemodynamically significant atherosclerotic renal artery stenosis and refractory systolic hypertension. Stent placement used distal protection. Optimal medical management included aggressive hypertension management, dyslipidemia management, diabetes, chronic kidney disease, smoking cessation, and anti-platelet drugs. Primary outcome for study was cardiovascular event or major renal dysfunction and death which related to cardiovascular event, renal dysfunction, stroke, myocardial infarction, hospitalization due to congestive heart failure, and the need of permanent renal replacement therapy. Primary outcome did not significantly differ between medical management group and stent group with hazard ratio 0.94 (35.8 vs $35.1 \%)$. Mortality rate was also not significantly different. Systolic blood pressure decreased in both groups with higher decrease in stent group (average $-2.3 \mathrm{mmHg}$ ). ${ }^{[30]}$

ASTRAL study ${ }^{[27]}$ compared revascularization therapy and optimal medical management. This study showed higher rate of renal dysfunction in medical therapy group with difference about $0.06 \times 10^{3} / \mathrm{L}$ every year. Average creatinine serum concentration was $1.6 \mu \mathrm{mol} / \mathrm{L}$ lower in patient with revascularization compared to medical therapy group. Systolic blood pressure was not significantly different between two groups but diastolic blood pressure had higher decrease in medical therapy group. But in this study, renal event (hazard ratio 0.97), cardiovascular event (hazard ratio 0.94 ), and mortality rate (hazard ratio 0.90) was equivalent between two groups.
In STAR study[30], authors observed the efficacy and safety of stent placement in patient with renal artery stenosis and decrease of renal function, compared to medical therapy management. Primary outcome in this study was the decrease of creatinine clearance $20 \%$ or more from baseline with two measurement. In stent group, if the primary outcome has been achieved, patient was sent for imaging examination to exclude re-stenosis of renal artery. Primary outcome was achieved in 22\% of medical therapy patient and $16 \%$ of stent patient in 10 months. 5 from 10 patients in stent group had repeated angioplasty before they could achieve primary outcome in which 2 of them had re-stenosis. This study also indicated there was not any significant difference for blood pressure control, cardiovascular morbidity and mortality, and incidence of worsening renal function. [31]

True renovascular hypertension occurred when hypertension caused by renal artery stenosis induced activation of renal angiotensin aldosterone system. This condition happened when the blood pressure did not improve after renal revascularization. It became special problem in diagnostic procedure because if the blood pressure did not improve after revascularization, patient might have renovascular hypertension or might be there were structural changes that prevent blood pressure from falling. Patient might also have essential hypertension accompanied by atherosclerotic renal artery stenosis with or without renovascular component. Renovascular hypertension could only happened in the condition of renal artery stenosis, but renal artery stenosis could occur without any renovascular hypertension.[3] Some studies showed failure of revascularization to improve clinical condition and anatomical diameter could not be used as 
prognostic factor in revascularization. $[19,20,21]$ Other studies used pressure gradient as predictor of significant stenosis in which fractional flow reserve $<0.80$ might be a good response predictor to revascularization.[31]

Gottam, et al. [1] mentioned that although renal artery stenosis was assumed to improve with revascularization and revascularization would also improve renovascular hypertension and nephropathy according to their pathophysiology, but some clinical studies did not always observe those same facts. Reasons behind those phenomena were complex and many studies is still on going to reveal many more of those reasons. Some process which could be related to hypertension after revascularization were sympathetic activation and endothelial dysfunction. ${ }^{[3]}$ Recent evidences indicated improvement of blood flow alone could not improve outcome of renal function and cardiovascular event in patient with renal artery stenosis.[11]

\section{O N C L U S I O N}

Current problem regarding renal artery stenosis was dilemma in diagnostic process and management. To date there was not any specific diagnostic criteria for renal artery stenosis. Some authors only suggest clinical suspicion which could possibly guide the diagnosis of renal artery stenosis. RADUS as one sensitive and specific procedure for diagnosing renal artery stenosis, sometimes was still confounded by some confounding factors. Imaging techniques such as CTA and MRA were excellent in diagnosing renal artery stenosis, but they were expensive and possessed the risk of ionized contrast and radiation exposure. To define severity of stenosis, clinician might use RADUS with and followed by CTA in some cases. But CTA was more recommended to performed in patient with planning of revascularization.

Clinical outcome for revascularization was best found in fibromuscular dysplasia with the using of balloon angioplasty, with success rate almost $100 \%$ with blood pressure control rate about $93 \%$. But complication rate of the procedure was still as high as $12 \%$ after angioplasty and $17 \%$ after surgery, that included arterial dissection, atheroembolism, and renal infarction. Some studies even showed equivalent effectivity of conservative medical therapy and angioplasty so the angioplasty was not recommended unless medical therapy had failed. In atherosclerosis, even though success rate of stenting achieved 95-100\%, but re-stenosis rate was $10-30 \%$ in 1 year.[1] Revascularization procedure could not entirely improve renovascular hypertension and nephropathy because they had complex mechanisms and improvement of renal blood flow alone could not ameliorate renal function nor cardiovascular event. From clinical studies, it was still recommended to choose revascularization in FMD, but revascularization should only be performed after the failure of optimal medical management in atherosclerotic renal artery stenosis

\section{R E F E R E N C E S}

1. Cho NH, Whiting D, Forouhi N, Guariguata L, Hambleton I, Li R, et al. IDF Diabetes Atlas In: Federation ID, editor. 2015.

2. Gottam N, Nanjundappa A, and Dieter RS. Renal artery stenosis: pathophysiology and treatment. Expert Rev.Cardiovasc. Ther. 2009.7 (11): 1413-1420. [doi: 10.1586/erc.09.109]

3. Weber BR, and Dieter RS. Renal artery stenosis: epidemiology and treatment. International Journal of Nephrology and Renovascular Disease, 2014; 7: 169181.

[doi: $10.2147 /$ IJNRD.S40175]

4. Bavishi C, de Leeuw PW, and Messerli FH. Atherosclerotic renal artery stenosis and hypertension: pragmatism, pitfalls, and perspectives. The American Journal of Medicine, 2016, 129: 635.e5-635.e14. [doi:https://doi.org/10.1016/i.amimed.2015.10.010]

5. Kasper DL, Hauser SL, Jameson JL, et al. Harrison's Principles of Internal Medicine 19th ed. USA: The McGraw-Hill Companies Inc. 2015. 
6. Parikh SA, Shishehbor MH, Gray BH, et al. SCAI Expert Consensus Statement for Renal Artery Stenting Appropriate Use. Catheterization and Cardiovascular Intervention, 2014, Wiley Online Library. [https://doi.org/10.1002/ccd.25559]

7. Goyez JCR, Gomez NIJ. Challenges in diagnosing and treating a patient with renal artery fibromuscular dysplasia: case report. European Heart Journal - Case Report, 2019, 3: 1-6. [doi:10.1093/ehjcr/yty144]

8. Al-Suraih M, Grande JP. Management of renal artery stenosis: What does the experimental evidence tell us? World J Cardiol, 2014, August 26; 6 (8): 855 - 860. [doi: 10.4330/wjc.v6.i8.855]

9. Walker BR, Colledge NR, Ralston, SH., et al. Davidson's Principles and Practice of Medicine 22nd ed. USA: Elsevier Inc. 2014.

10. Kumar, Parveen and Michael Clark. 2017. Kumar \& Clark's Clinical Medicine 9th ed. USA: Elsevier Inc.

11. Hall, John E. 2016. Guyton and Hall Textbook of Medical Physiology 13th ed. USA: Elsevier Inc.

12. Saragih, Wendy M. Case report of secondary hypertension due to renal artery stenosis in young patient. Med J Indones, 2014, 23 (2): 117-121. [doi:10.13181/mii.v23i2.666]

13. Colyer, WR., Eltahaway, E., Cooper, CJ. Renal artery stenosis: optimizing diagnosis and treatment. Prog Cardiovasc Dis, 2011; 54 (1): 29-35. [doi: 10.1016/i.pcad.2011.02.007]

14. McLaughlin, K., Jardine, AG., Moss, JG. ABC of Arterial and Venous Disease: Renal artery stenosis. BMJ Volume 320, April 2000.

[doi: https://doi.org/10.1136/bmj.320.7242.1124]

15. Taylor DC, Kettler MD, Moneta GL, et al. Duplex ultrasound scanning in the diagnosis of renal artery stenosis: a prospective evaluation. J. Vasc. Surgery, 1988, 7, 363-369. [doi:10.1016/0741-5214(88)90156-5]

16. Olin JW, Piedemonte MR, Young JR, et al. The utility of duplex ultrasound scanning of the renal arteries for diagnosing significant renal artery stenosis. Ann. Intern. Med. 1995. 122, 833-838. [doi: 10.7326/0003-4819122-11-199506010-00004]

17. Vasbinder, GBC, Nelemans PJ, Kessels AGH, et al. Accuracy of computed tomographic angiography and magnetic resonance angiography for diagnosing renal artery stenosis. Ann. Intern. Med. 2004. 141, 674-682. [https://doi.org/10.7326/0003-4819-141-9200411020-00007]

18. Postma CT, Joosten FB, Rosenbusch G et al. Magnetic resonance angiography has a high reliability in detection of renal artery stenosis. Am. J. Hypertens. 1997. 10, 957. [doi: 10.1016/s0895-7061(97)00157-x]

19. Williams GJ, Macaskill P, Chan SF, et al. Comparative accuracy of renal duplex sonographic parameters in the diagnosis of renal artery stenosis: paired and unpaired analysis. Am. J. Roentgenol, 2007. 188, 798. [doi: 10.2214/AJR.06.0355]

20. Textor S. Atherosclerotic renal artery stenosis: overtreated but underrated. J. Am. Soc. Nephrol. 2008. 19, 656-659.

[doi: https://doi.org/10.1681/ASN.2007111204]

21. Dieter R. The functional assessment of renal artery stenosis. Expert Rev. Cardiovasc. Ther. 2005. 3(3), 269370.

22. Jr Colyer WR, Cooper CJ, Burket MW, Thomas WJ. Utility of a 0.014 " pressure sensing guidewire to assess renal artery translesional systolic pressure gradients. Catheter Cardiovasc Interv. 2003. 59, 372-377. [doi: 10.1002/ccd.10508]
23. Safian RD, Madder RD. Refining the approach to renal artery revascularization. JACC Cardiovasc. Intervention, 2009. 2, 161-174.

[https://doi.org/10.1016/i.jcin.2008.10.014]

24. Wheatly K, Kalra PA, Moss J, etal. Lack of benefit of renal artery revascularization in atherosclerotic renovascular disease (ARVD). Results of the ASTRAL trial (abstr.). J. Am. Soc. Nephrol. 2008. 19, 656-659.

25. Cheung CM, Hegarty J, Kalra PA. Dilemmas in the management of renal artery stenosis. British Medical Bulletin, 2005, 73 \& 74: 35-55.

[https://doi.org/10.1093/bmb/ldh049]

26. Tegtmeyer CJ, Elson J, Glass TA, et al. Percutaneous transluminal angioplasty: the treatment of choice for renovascular hypertension due to fibromuscular dysplasia. Radiology, 1982. 143, 631-637. [doi: 10.1148/radiology.143.3.6210930 ]

27. Rocha-Singh K, Michael RJ, Kenneth R. Evaluation of the Safety and Effectiveness of Renal Artery Stenting after Unsuccessful Balloon Angioplasty: The ASPIRE-2 Study. Journal of the American College of Cardiology, 2005, 46, 5: 776-783. [https://doi.org/10.1016/i.jacc.2004.11.073

28. The ASTRAL Investigators. Revascularization versus Medical Therapy for Renal Artery Stenosis. N Engl J Med, 2009; 361: 1953-1962. [doi: 10.1056/NEJMoa0905368]

29. Murphy TP, Cooper CJ, Dworkin LD, et al. The Cardiovascular Outcomes with Renal Atherosclerotic Lesions (CORAL) Study: Rationale and Methods. J Vasc Interv Radiol, 2005; 16: 1295-1300. [doi:https://doi.org/10.1097/01.RVI.0000176301.697 $\underline{56.28}$

30. Leertouwer TC, Gussenhoven EJ, Bosch JL, et al. Stent placement for renal arterial stenosis: where do we stand? A meta-analysis. Radiology 2000; 216:78-85. [doi: 10.1148/radiology.216.1.r00j10778]

31. Cooper CJ, Murphy TP, Cutlip DE, et al. 2014. Stenting and Medical Therapy for Atherosclerotic Renal-Artery Stenosis. N Eng J Med, 2014, 370: 13-22. [doi: 10.1056/NEJMoa1310753]

32. Bax L, Woittiez AJ, Kouwenberg HJ, et al. Stent Placement in Patients with Atherosclerotic Renal Artery Stenosis and Impaired Renal Function. A Randomized Trial. Ann Intern Med, 2009; 150: 840-848. [doi: 10.7326/0003-4819-150-12-200906160-00119]

\section{Cite this as}

Waafi AK, Samsu N. Renal Artery Stenosis: Diagnostic and Management Problems. Clinical and Research Journal in Internal Medicine. 1.2 (2020): 96-110 